\title{
A SHORT PROOF OF THE HERMITE'S FORMULA FOR POLYNOMIAL INTERPOLATION USING OPERATOR METHODS
}

\author{
BY
}

DANIEL STĂNICĂ

\begin{abstract}
In this paper we present a short proof of the Hermite's formula for polynomial interpolation using the theory of linear algebra, without using Taylor series expansion as in the classic proof of this formula. In this construction we use a schema of interpolation defined by the inverse of a matrix.
\end{abstract}

Mathematics Subject Classification 2010: 65D05, 65D15, 15 A09.

Key words: Hermites's formula, polynomial interpolation, interpolation scheme.

The Hermite's formula for polynomial interpolation is well known and it is successfully used in practice. It is described in many books of numerical analysis, e.g. $[2,3,8]$. Our goal is to offer a way to build the Hermite's interpolation polynomial and Hermite's formula for its representation. Based on the idea from [1], which is extended in an interpolation scheme in [4], we take a certain base in the space of polynomials of degree at most $n-1 \in \mathbf{N}$. Then with the interpolation scheme, we represent the Hermite interpolation polynomial and we obtain the Hermite's formula.

Further, we consider a particular case of this schema. Let $n \in \mathbf{N}^{*}, X$ be a vector space, $Y$ a $n$-dimensional subspace of $X$ and $U: X \rightarrow \mathbf{R}^{n}$ a linear operator. We denote by $V: \mathbf{R}^{n} \rightarrow Y$ an isomorphism between the two spaces. We assume that the operator $U V: \mathbf{R}^{n} \rightarrow \mathbf{R}^{n}$ is invertible.

We define the operator $P_{U}: X \rightarrow Y$ by $P_{U}=V(U V)^{-1} U$ and we call it an interpolation operator of $X$ by elements of $Y$, relative to the operator $U$. 
Lemma 1. The following statements are true:

a) $U(x)=U P_{U}(x)$, for all $x \in X$;

b) $P_{U}(y)=y$, for all $y \in Y$;

c) Let $x \in X$. If $y_{0} \in Y$ verifies $U(x)=U\left(y_{0}\right)$, then $y_{0}=P_{U}(x)$.

Proof. a) We have $U(x)-U P_{U}(x)=U(x)-U V(U V)^{-1} U(x)=0$.

b) The operator $U$ restricted to $Y$ is injective. Indeed, let us consider $y, z \in Y$ with $U(y)=U(z)$ and $y_{1}, z_{1} \in \mathbf{R}^{n}$ such that $y=V\left(y_{1}\right)$ and $z=V\left(z_{1}\right)$. It results that $U V\left(y_{1}\right)=U V\left(z_{1}\right)$ and because $U V$ is bijective we deduce that $y_{1}=z_{1}$, and because $V$ is bijective we obtain $y=z$.

From a) it results that $U(y)-U P_{U}(y)=0$ and from the fact that $U$ is injective we obtain $y=P_{U}(y)$.

c) We have $U\left(P_{U}(x)\right)=U(x)=U\left(y_{0}\right)$ and because restriction of $U$ at $Y$ is injective, it results that $y_{0}=P_{U}(x)$.

We consider the next data system (noted by $(\mathrm{SH})$ ):

- $n \in \mathbf{N}^{*}$

- the points $x_{1}, x_{2}, \ldots, x_{n} \in[a, b] \subset \mathbf{R}$;

- $m \in \mathbf{N}^{*}$ the number of distinct points;

- $y_{1}, y_{2}, \ldots, y_{m} \in \mathbf{R}$ the distinct points;

- $n_{1}, n_{2}, \ldots, n_{m} \in \mathbf{N}^{*}$ the multiplicity order of distinct points $\left(n_{1}+n_{2}+\right.$ $\left.\ldots+n_{m}=n\right)$.

Let $X=\left\{f:[a, b] \rightarrow \mathbf{R} \mid f\right.$ is $n_{i}-1$-times differentiable at $y_{i}$, for all $i \in \overline{1, m}\}, Y=\mathcal{P}_{n-1}$. Let $U: X \rightarrow \mathbf{R}^{n}$ be defined by

$$
U f=\left(f\left(y_{1}\right), f^{\prime}\left(y_{1}\right), \ldots, f^{\left(n_{1}-1\right)}\left(y_{1}\right), \ldots, f\left(y_{m}\right), f^{\prime}\left(y_{m}\right), \ldots, f^{\left(n_{m}-1\right)}\left(y_{m}\right)\right)
$$

and let $V$ be the canonical isomorphism between $\mathbf{R}^{n}$ and $\mathcal{P}_{n-1}$. If we consider the canonical basis $e_{1}, e_{2}, \ldots, e_{n}$ in $\mathbf{R}^{n}$, then the operator $U V$ has the following matrix representation

$$
U V=\left(\begin{array}{c}
M_{1} \\
M_{2} \\
\cdots \\
M_{m}
\end{array}\right),
$$


where

$$
M_{j}=\left(\begin{array}{ccccc}
1 & y_{j} & y_{j}^{2} & \ldots & y_{j}^{n-1} \\
0 & 1 & 2 y_{j} & \ldots & (n-1) y_{j}^{n-2} \\
0 & 0 & 2 & \ldots & (n-1)(n-2) y_{j}^{n-3} \\
\vdots & \vdots & \vdots & \ddots & \vdots \\
0 & 0 & 0 & \ldots & A_{n-1}^{n_{j}-1} y_{j}^{n-n_{j}}
\end{array}\right), \forall j=\overline{1, m}
$$

Since the determinant of this matrix is $\prod_{i=1}^{m}\left(\prod_{j=0}^{n_{i}-1} j !\right) \prod_{1 \leq i<j \leq m}\left(y_{j}-\right.$ $\left.y_{i}\right)^{n_{i} n_{j}} \neq 0$ (see [5]), then there exists $(U V)^{-1}$.

Let $P_{U}: X \rightarrow Y, P_{U}=V(U V)^{-1} U$ be the interpolation operator of $X$ by elements of $Y$, relative to the operator $U$. For $f \in X$ the element $P_{U}(f) \in \mathcal{P}_{n-1}$ is the Hermite polynomial attached to the function $f$ and to the data system $(S H)$.

Using Lemma 1 it results that $U(f)=U P_{U}(f)$, so $P_{U}(f)$ is the unique real $n-1$ degree polynomial which has the property

$$
\left(P_{U}(f)\right)^{(j)}\left(y_{i}\right)=f^{(j)}\left(y_{i}\right), \forall j=\overline{0, n_{i}-1}, \forall i=\overline{1, m} .
$$

Further, using the theory of linear algebra, we are proving the Hermite representation formula for the Hermite polynomial interpolation.

Lemma 2. Let $\omega(x)=\prod_{i=1}^{m}\left(x-y_{i}\right)^{n_{i}}$. Then the family of polynomials $p_{i j}(x)=\frac{\omega(x)}{\left(x-y_{i}\right)^{n_{i}-j}}, j=\overline{0, n_{i}-1}, i=\overline{1, m}$ forms a basis in the space $\mathcal{P}_{n-1}$.

Proof. It is enough to show that the polynomials $\left(p_{i j}\right)_{j=\overline{0, n_{i}-1}}, i=\overline{1, m} \subset$ $\mathcal{P}_{n-1}$ are linearly independent. Let us consider $\left(a_{i j}\right)_{j=\overline{0, n_{i}-1}, i=\overline{1, m}} \subset \mathbf{R}$ such that

$$
\sum_{i=1}^{m} \sum_{j=0}^{n_{i}-1} a_{i j} p_{i j}(x)=0 .
$$

We have $p_{i j}^{(l)}\left(y_{k}\right)=0$ if $k \neq i$ or $k=i$ and $l \neq j$, and $p_{i j}^{(j)}\left(y_{i}\right) \neq 0$. Let $i \in \overline{1, m}$. Then taking $x=y_{i}$ in (1) it results that $a_{i 0}=0$. We consecutively derive the same equality up to the order $n_{i}-1$ and taking $x=y_{i}$ we obtain $a_{i j}=0$ for $j=\overline{0, n_{i}-1}$. Hence $a_{i j}=0, \forall j=\overline{0, n_{i}-1}, \forall i=\overline{1, m}$.

We consider $V$ the isomorphism between $\mathbf{R}^{n}$ and $\mathcal{P}_{n-1}$ given by

$$
V\left(\left(a_{i j}\right)_{j=\overline{0, n_{i}-1}} i=\overline{1, m}\right)=\sum_{i=1}^{m} \sum_{j=0}^{n_{i}-1} a_{i j} h_{i j}(x) .
$$


If we take $e_{1}=(1,0, \ldots, 0), e_{2}=(0,1, \ldots, 0), \ldots, e_{n}=(0,0, \ldots, 1)$ the canonical basis in $\mathbf{R}^{n}$, the operator $U V$ has the following blocks-diagonal matrix representation:

$$
U V=\left(\begin{array}{cccc}
M_{1} & 0 & \ldots & 0 \\
0 & M_{2} & \ldots & 0 \\
\vdots & \vdots & \ddots & \vdots \\
0 & 0 & \ldots & M_{m}
\end{array}\right)
$$

where each matrix $M_{i}$ is square, lower triangular, with $n_{i}$-dimension, i.e.

$$
M_{i}=\left(\begin{array}{cccc}
\left.\frac{\omega(x)}{\left(x-y_{i}\right)^{n_{i}}}\right|_{x=y_{i}} & 0 & \cdots & 0 \\
\left(\frac{\omega(x)}{\left(x-y_{i}\right)^{n_{i}}}\right)_{x=y_{i}}^{\prime} & \left(\frac{\omega(x)}{\left(x-y_{i}\right)^{n_{i}-1}}\right)_{x=y_{i}}^{\prime} & \cdots & 0 \\
\vdots & \vdots & \ddots & \vdots \\
\left(\frac{\omega(x)}{\left(x-y_{i}\right)^{n_{i}}}\right)_{x=y_{i}}^{\left(n_{i}-1\right)} & \left(\frac{\omega(x)}{\left(x-y_{i}\right)^{n_{i}-1}}\right)_{x=y_{i}}^{\left(n_{i}-1\right)} & \cdots & \left(\frac{\omega(x)}{\left(x-y_{i}\right)}\right)_{x=y_{i}}^{\left(n_{i}-1\right)}
\end{array}\right),
$$

for all $i=\overline{1, m}$. Let $j \in \overline{0, k}$ with $k \in \overline{0, n_{i}-1}$. We have

$$
\left(\frac{\omega(x)}{\left(x-y_{i}\right)^{n_{i}-j}}\right)_{x=y_{i}}^{(k)}=\left(\left(x-y_{i}\right)^{j} \frac{\omega(x)}{\left(x-y_{i}\right)^{n_{i}}}\right)_{x=y_{i}}^{(k)}=j ! C_{k}^{j}\left(\frac{\omega(x)}{\left(x-y_{i}\right)^{n_{i}}}\right)_{x=y_{i}}^{(k-j)} .
$$

If we denote $\frac{\omega(x)}{\left(x-y_{i}\right)^{n_{i}}}$ by $g_{i}(x)$, then

$$
M_{i}=\left(\begin{array}{ccccc}
g_{i}\left(y_{i}\right) & \ldots & 0 & \ldots & 0 \\
\vdots & \ddots & \vdots & \ddots & \vdots \\
g^{(k)}\left(y_{i}\right) & \vdots & j ! C_{k}^{j} g_{i}^{(k-j)}\left(y_{i}\right) & \ldots & 0 \\
\vdots & \ddots & \vdots & \ddots & \vdots \\
g^{\left(n_{i}-1\right)}\left(y_{i}\right) & \ldots & j ! C_{n_{i}-1}^{j} g_{i}^{\left(n_{i}-j-1\right)}\left(y_{i}\right) & \ldots & \left(n_{i}-1\right) ! g_{i}^{\left(n_{i}-1\right)}\left(y_{i}\right)
\end{array}\right)
$$

Lemma 3. The inverse of the matrix $U V$ is

$$
(U V)^{-1}=\left(\begin{array}{cccc}
M_{1}^{-1} & 0 & \ldots & 0 \\
0 & M_{2}^{-1} & \ldots & 0 \\
\vdots & \vdots & \ddots & \vdots \\
0 & 0 & \ldots & M_{m}^{-1}
\end{array}\right)
$$


with $M_{i}^{-1}=$

$$
=\left(\begin{array}{ccccc}
h_{i}\left(y_{i}\right) & \ldots & 0 & \ldots & 0 \\
\vdots & \ddots & \vdots & \ddots & \vdots \\
\frac{1}{k !} h^{(k)}\left(y_{i}\right) & \vdots & \frac{C_{k}^{j}}{k !} h_{i}^{(k-j)}\left(y_{i}\right) & \ldots & 0 \\
\vdots & \ddots & \vdots & \ddots & \vdots \\
\frac{1}{\left(n_{i}-1\right) !} h^{\left(n_{i}-1\right)}\left(y_{i}\right) & \ldots & \frac{C_{n_{i}-1}^{j}}{\left(n_{i}-1\right) !} h_{i}^{\left(n_{i}-j-1\right)}\left(y_{i}\right) & \ldots & \frac{1}{\left(n_{i}-1\right) !} h_{i}\left(y_{i}\right)
\end{array}\right) \text {, }
$$

where $j \leq k$ and $k \in \overline{0, n_{i}-1}$ and $h_{i}(x)=\frac{1}{g_{i}(x)}, \forall i=\overline{1, m}$.

Proof. Since the matrix $U V$ is blocks-diagonal, then its inverse matrix is blocks-diagonal. Let $j \leq k$ with $k \in \overline{0, n_{i}-1}$. Multiplying the $k$-row of the matrix $M_{i}$ with the $j$-column of the matrix $M_{i}^{-1}$ we obtain the value

$$
\begin{aligned}
& \sum_{p=j}^{k} p ! C_{k}^{p} g_{i}^{(k-p)}\left(y_{i}\right) \frac{C_{p}^{j}}{p !} h_{i}^{(p-j)}\left(y_{i}\right)=C_{k}^{j} \sum_{p=j}^{k} C_{k-j}^{p-j} g_{i}^{(k-p)}\left(y_{i}\right) h_{i}^{(p-j)}\left(y_{i}\right)= \\
& =C_{k}^{j} \sum_{s=0}^{k-j} C_{k-j}^{s} g_{i}^{(k-j-s)}\left(y_{i}\right) h_{i}^{(s)}\left(y_{i}\right)=C_{k}^{j}\left(g_{i} h_{i}\right)^{(k-j)}\left(y_{i}\right)=\left\{\begin{array}{ll}
0, & \text { if } j \neq k \\
1, & \text { if } j=k
\end{array},\right.
\end{aligned}
$$

which proves that $M_{i} M_{i}^{-1}=\mathbf{I}_{n_{i}}$, hence $(U V)^{-1}$ takes the form claimed $i$ the statement of this lemma.

Theorem 4. The Hermite interpolation polynomial attached to the function $f$ and to the data system $(S H)$ admits the following representation formula

$$
\begin{aligned}
P_{U}(f) & =\sum_{i=1}^{m} \frac{\omega(x)}{\left(x-y_{i}\right)^{n_{i}}} \\
& \cdot\left[\sum_{j=0}^{n_{i}-1} f^{(j)}\left(y_{i}\right) \frac{\left(x-y_{i}\right)^{j}}{j !}\left(\sum_{k=0}^{n_{i}-j-1} \frac{\left(x-y_{i}\right)^{k}}{k !}\left(\frac{\left(x-y_{i}\right)^{n_{i}}}{\omega(x)}\right)_{x=y_{i}}^{(k)}\right)\right]
\end{aligned}
$$

Proof. Using the basis from Lemma 2 and the operator $V$ defined in (2), it results that

$$
\begin{aligned}
P_{U}(f) & =V(U V)^{-1} U(f) \\
& =\sum_{i=1}^{m} \sum_{j=0}^{n_{i}-1}\left(\sum_{k=j}^{n_{i}-1} \frac{\omega(x)}{\left(x-x_{i}\right)^{n_{i}-k}} \frac{C_{k}^{j}}{k !} h_{i}^{(k-j)}\left(y_{i}\right)\right) f^{(j)}\left(y_{i}\right)
\end{aligned}
$$




$$
\begin{aligned}
& =\sum_{i=1}^{m} \sum_{j=0}^{n_{i}-1}\left(\sum_{k=0}^{n_{i}-j-1} \frac{\omega(x)}{\left(x-x_{i}\right)^{n_{i}-k-j}} \frac{1}{j ! k !}\left(\frac{\left(x-y_{i}\right)^{n_{i}}}{\omega(x)}\right)_{x=y_{i}}^{(k)}\right) f^{(j)}\left(y_{i}\right) \\
& =\sum_{i=1}^{m} \frac{\omega(x)}{\left(x-y_{i}\right)^{n_{i}}} \\
& \cdot\left[\sum_{j=0}^{n_{i}-1} f^{(j)}\left(y_{i}\right) \frac{\left(x-y_{i}\right)^{j}}{j !}\left(\sum_{k=0}^{n_{i}-j-1} \frac{\left(x-y_{i}\right)^{k}}{k !}\left(\frac{\left(x-y_{i}\right)^{n_{i}}}{\omega(x)}\right)_{x=y_{i}}^{(k)}\right)\right] .
\end{aligned}
$$

For example, let $m=2, y_{1}=2, y_{2}=3, n_{1}=1$ and $n_{2}=3$. The basis from Lemma 2 for $\mathcal{P}_{3}$ is $p_{10}(x)=x^{3}-9 x^{2}+27 x-27, p_{20}(x)=x-2$, $p_{21}(x)=x^{2}-5 x+6, p_{22}(x)=x^{3}-8 x^{2}+21 x-18$. In this basis we have $1=$ $-p_{10}(x)+p_{20}(x)-p_{21}(x)+p_{22}(x), x=-2 p_{10}(x)+3 p_{20}(x)-2 p_{21}(x)+2 p_{22}(x)$, $x^{2}=-4 p_{10}(x)+9 p_{20}(x)-3 p_{21}(x)+4 p_{22}(x)$ and $x^{3}=-8 p_{10}(x)+27 p_{20}(x)+$ $9 p_{22}(x)$.

Remark 1. A similar technique of obtaining a representation of the Hermites interpolation polynomials is presented in $[6,7]$, using the spectral basis. By choosing the basis of Lemma 2 we obtain an explicit representation formula. It represents a real advantage because such a formula is not found in the above-mentioned papers.

Remark 2. In a similar way Hermite's formula can be extended to the exponential and trigonometric interpolation.

\section{REFERENCES}

1. DE Boor, C. - What is the inverse of a basis?, BIT 40th Anniversary Meeting. BIT, 41 (2001), suppl., 880-890.

2. IChim, I.; MARinescu, G. - Numerical Approximation Methods (in romanian), Editura Academiei Române, 1987.

3. Phillips, G.M. - Interpolation and Approximation by Polynomials, CMS Books in Mathematics/Ouvrages de Mathématiques de la SMC, 14, Springer-Verlag, New York, 2003. 
4. STĂNICĂ, D. - An interpolation scheme in the topological vector space defined using the pseudoinverse, Mathematical Reports, 7 (2005), 325-333.

5. SoвcZYк, G. - Generalized Vandermonde determinants and applications, XXXIV National Congress of the Mexican Mathematical Society (Spanish) (Toluca, 2001), 203-213, Aportaciones Mat. Comun., 30, Soc. Mat. Mexicana, México, 2002.

6. Soвczyк, G. - The missing spectral basis in algebra and number theory, Amer. Math. Monthly, 108 (2001), 336-346.

7. SobczyK, G. - Quantum Hermite interpolation polynomials, Parametric optimization and related topics. VII, 105-112, Aportaciones Mat. Investig., 18, Soc. Mat. Mexicana, México, 2004.

8. J.F. Steffensen - Interpolation, Second Edition, Dover Publications, 2006.

Received: 14.III.2012

Revised: 27.III.2012

Accepted: 3.IV.2012
University of Bucharest, Faculty of Mathematics and Computer Science, Department of Mathematics, Str Academiei No. 14, 010014, Bucharest, ROMANIA

stanicad@fmi.unibuc.ro 